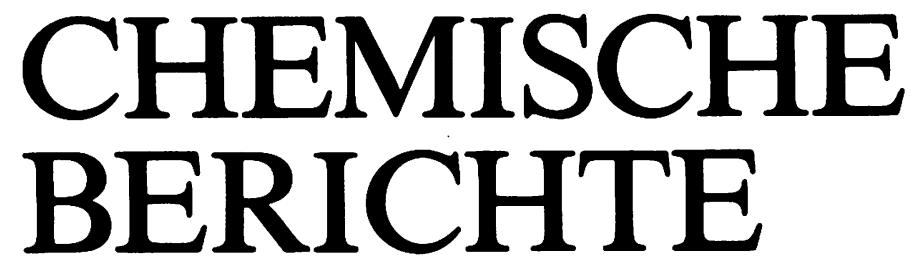

GEGRÜNDET 1868

115. JAHRGANG

HERAUSGEGEBEN IM AUFTRAG DER

GESELLSCHAFT DEUTSCHER CHEMIKER

VON

K. HAFNER - W. KIRMSE - H. MUSSO - H. NÖTH • J. SAUER - E. WINTERFELDT

UNTER MITWIRKUNG VON

H. A. BRUNE · W. LÜTTKE · G. SPITELLER

REDAKTION: H. ZAHN

mit H. SCHILL, J. STREHLOW und A. WIELAND 


\section{PHYSICAL CHEMISTRY}

Hellmann Goetz, Hellmann Siegried, Beckhaus Hans-Dieter, and Rüchardt Christoph: Thermolabile Hydrocarbons, XVI: Thermal Stability, Strain Enthalpy, and Struc-

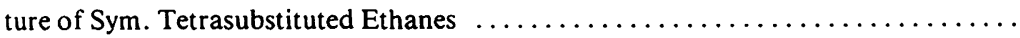

\section{INORGANIC CHEMISTRY}

Richter Felix and Vahrenkamp Heinrich: Chiral SFeCoM Clusters: Synthesis, Side

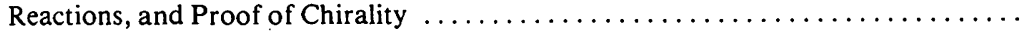

Richter Felix and Vahrenkamp Heinrich: Chiral SFeCoM Clusters: Enantiomer Separation and Determination of the Absolute Configuration

Keçeci Ahmet, Rehder Dieter, Roose Wolfgang, and Talay Ridvan: Phosphane-bridged Dinuclear Complexes Containing Carbonyl- $\eta^{5}$-cyclopentadienyl Transition Metal

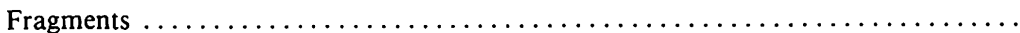

Maringgele Walter: Reaction of Metal and Metalloid Compounds with Polyfunctional Molecules, XXXVI: New Syntheses of Open-Chain and Cyclic $\mathrm{N}$-Borylureas and

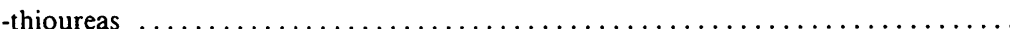

Schmidbaur Hubert, Deschler Ulrich, and Milewski-Mahrla Beatrix: Ylide Complexes of Alkali and Alkaline Earth Metals, V: Pyridine-substituted Phosphonium-bis-methylide Anions as Chelating Ligands for Sodium, Potassium, and Barium. Crystal Structure of a Binuclear Potassium Complex ........................

Sohn Dieter and Sundermeyer Wolfgang: Reaction of Pentafluoro-2-aza-1-propene with Perfluorinated Carbenes . . . . . . . . . . . . . . . . . . . . . . . . . .

Köster Hajo and Weiss Erwin: Metal Alkyl and Aryl Compounds, XXVIII: Preparation and Crystal Structure of Allyllithium- $N, N, N^{\prime}, N^{\prime}$-Tetramethylethylenediamine,



Weidenbruch Manfred, Flott Hermann, Fleischhauer Jörg, and Schleker Wolfgang: Silicon Compounds with Strong Intramolecular Steric Interactions, 13: Restricted Rotations in Tri-tert-butylsilanes

\section{ORGANIC CHEMISTRY}

Leininger Hartmut, Kemmer Petra, Beck Karin, and Christl Manfred: 7-Thiatetracyclo$\left[4.1 .0 .0^{2,4} \cdot 0^{3,5}\right]$ heptane (Benzvalene Sulfide) - Synthesis and Reactions . . . . . . .

Maringgele Walter: Reaction of Metal and Metalloid Compounds with Polyfunctional Molecules, XXXVI: New Syntheses of Open-Chain and Cyclic $N$-Borylureas and -thioureas

Erker Gerhard, Wicher Joachim, Engel Klaus, and Krüger Carl: (s-trans- $\eta^{4}$-Diene)zirconocene Complexes

Erker Gerhard, Engel Klaus, Krüger Carl, and Chiang An-Pei: Reactivity and Structure of (s-cis-1,3-Diene)zirconocene Complexes

Roedig Alfred and Ritschel Werner: Reactions of 3,4,4-Trichloro-3-butenamides with Nucleophiles, I: Dimers and Alcohol Adducts of 1,1-Dichloroallenecarboxamides .. 
Schaumann Ernst, Bäuch Hans-Günther, Sieveking Stefan, and Adiwidjaja Gunadi: Cycloaddition Reactions of Heterocumulenes, XXV: Cycloadducts and Rearrangement Products from the Reaction of Isothiocyanates with Ketene Acetals ..........

Neidlein Richard and Zeiner Hartmut: Syntheses of Some Unsaturated Carboxylic Acids of 1,6-Methano[10]annulene and the Bridged Dicyanopseudophenalene-fulvene ....

Hellmann Goetz, Hellmann Siegried, Beckhaus Hans-Dieter, and Rüchardt Christoph: Thermolabile Hydrocarbons, XVI: Thermal Stability, Strain Enthalpy, and Structure of Sym. Tetrasubstituted Ethanes $\ldots \ldots \ldots \ldots \ldots \ldots \ldots \ldots \ldots \ldots$

Heuschmann Manfred and Quast Helmut: Three-membered Ring Heterocycles, 14: $\alpha$-Halogenation of Tertiary Phosphane Oxides $\ldots \ldots \ldots \ldots \ldots \ldots \ldots \ldots$

Auchter Gerhard and Hanack Michael: Vinyl Cations, 38: Synthesis and Solvolysis of 3-Substituted 1-Cyclobutenyl Nonaflates $\ldots \ldots \ldots \ldots \ldots \ldots \ldots \ldots \ldots \ldots \ldots$

Cetinkaya Bekir, Binger Paul, and Krüger Carl: Metallacycloalkanes, V: Preparation of a Rhodacycloheptane by Oxidative Coupling of 3,3-Dimethylcyclopropene to

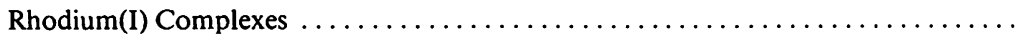

Welt Günther, Wolf Elisabeth, Fischer Peter, and Föhlisch Baldur: [4+2] Cycloadducts with Alternate Structure from 7,7-Difluoro- and 7,7-Dialkoxy-1,3,5-cycloheptatrienes and 4-Phenyl-1,2,4-triazoline-3,5-dione $\ldots \ldots \ldots \ldots \ldots \ldots \ldots \ldots \ldots$

Ruminski Jan K. and Przewoska Krystyna D.: Synthesis and Reactivity of 2-Aroylbenzoic Acids, II: 2-(4-Hydroxy-3-isopropylbenzoyl)benzoic Acid ...............

Weidenbruch Manfred, Flott Hermann, Fleischhauer Jörg, and Schleker Wolfgang: Silicon Compounds with Strong Intramolecular Steric Interactions, 13: Restricted Rotations in Tri-tert-butylsilanes

Zander Maximilian: Reaction of Carbazolylpotassium with 9-Bromoanthracene and Nitrobenzene 


\section{AUTORENREGISTER}

Adiwidjaja, G. s. Schaumann, E. . . . 3340

Auchter, G. und Hanack, M. ....... 3402

Bäuch, H.-G. s. Schaumann, E. . . . . 3340

Beck, K. s. Leininger, H. . . . . . . 3213

Beckhaus, H.-D. s. Hellmann, G. . . . 3364

Binger, P. s. Cetinkaya, B. ....... 3414

Cetinkaya, B., Binger, $P$. und

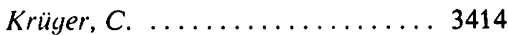

Chiang, A.-P. s. Erker, G. . ...... 3311

Christl, M. s. Leininger, H. . . . . . 3213

Deschler, U. s. Schmidbaur, H. . . . . 3290

Engel, K. s. Erker, G. . . . . . . 3300, 3311

Erker, G., Engel, K., Krüger, C. und

Chiang, A.-P. . .......... 3311

-, Wicher, J., Engel, K. und

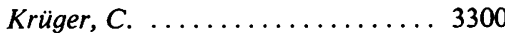

Fischer, P. s. Welt, G. .......... 3427

Fleischhauer, J. s. Weidenbruch, M. .. 3444

Flott, $H$. s. Weidenbruch, $M$. . . . . . 3444

Föhlisch, B. s. Welt, G. . . . . . . . 3427

Hanack, M. s. Auchter, G. . . . . . . 3402

Hellmann, G., Hellmann, S.,

Beckhaus, H.-D. und Rüchardt, C. 3364

Hellmann, S. s. Hellmann, G. . . . . . 3364

Heuschmann, M. und Quast, H. . ... 3384

Keçeci, A., Rehder, D., Roose, W.

und Talay, $R . \ldots \ldots \ldots \ldots \ldots 257$

Kemmer, P. s. Leininger, H. . . . . . 3213

Köster, H. und Weiss, E. . . . . . . . 3422

Krüger, C. s. Cetinkaya, B. . . . . . . 3414

- s. Erker, G. . . . . . . . . . 3300, 3311

Leininger, H., Kemmer, P., Beck, $K$.

und Christl, $M$.

3213
Maringgele, $W . \ldots \ldots \ldots \ldots \ldots . \ldots . .3271$

Milewski-Mahrla, B. s. Schmidbaur, H. 3290

Neidlein, $R$. und Zeiner, H. . . . . 3353

Przewoska, K. D. s. Rumiński, J. K. .. 3436

Quast, H. s. Heuschmann, M. . . . . . 3384

Rehder, D. s. Keçeci, A. . . . . . . . 3257

Richter, F. und Vahrenkamp, H. . 3224, 3243

Ritschel, W. s. Roedig, A. . . . . . . 3324

Roedig, A. und Ritschel, W. . . . . . 3324

Roose, W. s. Keçeci, A. . . . . . . 3257

Rüchardt, C. s. Hellmann, G. . . . . . 3364

Rumiński, J. K. und Przewoska, K. D. . 3436

Schaumann, E., Bäuch, H.-G.,

Sieveking, S. und Adiwidjaja, G. . 3340

Schleker, W. s. Weidenbruch, M. . . . . 3444

Schmidbaur, $H .$, Deschler, $U$. und

Milewski-Mahrla, B. . ........ 3290

Sieveking, S. s. Schaumann, E. . . . . 3340

Sohn, D. und Sundermeyer, W. . . . . 3334

Sundermeyer, W. s. Sohn, D. . ...... 3334

Talay, R. s. Keçeci, A. . . . . . . . . 3257

Vahrenkamp, H. s. Richter, F. . . . 3224, 3243

Weidenbruch, M., Flott, H.,

Fleischhauer, J. und Schleker, W. . 3444

Weiss, E. s. Köster, H. . . . . . . 3422

Welt, G., Wolf, E., Fischer, P. und

Föhlisch, B. . . . . . . . . . 3427

Wicher, J. s. Erker, G. . . . . . . . 3300

Wolf, E. s. Welt, G. . . . . . . . . 3427

Zander, M. ............... 3449

Zeiner, $H$. s. Neidlein, $R . \quad \ldots \ldots \ldots \ldots 3353$ 


\title{
Ab-initio-MO-Studie Methyl- und Phenyl-substituierter Allenyl-Kationen
}

\author{
Herbert Mayr* und Reinhard Schneider \\ Institut für Organische Chemie der Universität Erlangen-Nürnberg, \\ Henkestr. 42, D-8520 Erlangen
}

Eingegangen am 1. März 1982

An den Methyl- und Phenyl-substituierten Allenyl-Kationen 3-12 (Tab. 1) wurden ab-initioMO-Berechnungen unter Verwendung des STO-3G Basissatzes durchgeführt. Die berechneten Bindungslängen und Ladungsverteilungen zeigen Delokalisierung der positiven Ladung an, wie in Formel 1 gezeigt. Mit Hilfe isodesmischer Reaktionen werden Stabilisierungsenergien von Substituenten in 1- und 3-Position ermittelt. Diese Werte ermöglichen in Kombination mit der experimentell bekannten Bildungswärme des Stammkörpers 2 die Bestimmung von $\Delta H_{\mathrm{f}}^{\circ}$ sämtlicher Allenyl-Kationen 3-12. Der Vergleich dieser Daten mit einigen experimentell bestimmten Bildungswärmen zeigt Übereinstimmung innerhalb von $2 \mathrm{kcal} / \mathrm{mol}$. Es werden Voraussagen für das Reaktionsverhalten gegenüber $\mathrm{n}$-Nucleophilen und $\pi$-Systemen gemacht.

\section{Ab initio MO Study of Methyl and Phenyl Substituted Allenyl Cations}

$\mathrm{Ab}$ initio MO calculations at the STO-3G basis set level have been performed for the methyl and phenyl substituted allenyl cations 3-12 (Table 1). The calculated bond lengths and charge distributions indicate delocalization of the positive charge as indicated in formula 1. Stabilization energies of substituents in 1-and 3-position are derived on the basis of isodesmic reactions. These values and the experimentally known heat of formation of the parent allenyl cation 2 allow evaluation of $\Delta H_{\mathrm{f}}^{\mathrm{o}}$ of all allenyl cations $3-\mathbf{1 2}$. Comparison of these data with some heats of formation from experimental sources show deviations of less than $2 \mathrm{kcal} / \mathrm{mol}$. Finally, predictions for the reactivity of allenyl cations with n-nucleophiles and $\pi$-systems are made.

Allenyl- bzw. Propargyl-Kationen 1 lassen sich sowohl als konjugierte Vinyl-Kationen wie auch als ungesättigte Allyl-Kationen beschreiben.

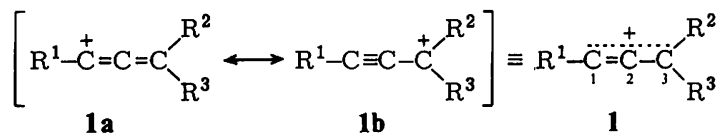

Sie wurden als reaktive Zwischenstufen bei der Solvolyse von Allenyl- und Propargyl-Derivaten nachgewiesen und als stabile Spezies in superaciden Medien beobachtet ${ }^{1-5}$ ). Ihre Additions- und Cycloadditionsreaktionen mit Olefinen und Dienen wurden von uns in den letzten Jahren untersucht ${ }^{6}$.

Frühere theoretische Arbeiten befaßten sich mit der Ladungsverteilung und Struktur Methylund Fluor-substituierter Allenyl-Kationen ${ }^{7-9)}$.

(C) Verlag Chemie GmbH, D-6940 Weinheim, 1982

$0009-2940 / 82 / 1111-3470 \$ 02.50 / 0$ 
Energetische Aspekte blieben bei diesen Studien unberücksichtigt. Wir ergänzen diese Untersuchungen nun durch Phenyl-substituierte Systeme, die für unsere experimentellen Arbeiten ${ }^{6}$ ) von Interesse sind, und präsentieren erstmals quantitative Daten über die Stabilität unterschiedlich substituierter Allenyl-Kationen.

\section{Methode}

Die Bestimmung zuverlässiger Energieunterschiede verschiedener Carbenium-Ionen mit Hilfe von ab-initio-MO-Methoden erfordert üblicherweise die Verwendung eines großen Basissatzes ${ }^{10}$. Derzeit können damit jedoch nur relativ kleine Teilchen analysiert werden. Am Beispiel von Allyl- ${ }^{11)}$ und Vinyl-Kationen ${ }^{12)}$ haben wir allerdings demonstriert, daß schon Rechnungen mit dem minimalen STO-3G-Basissatz ${ }^{13)}$ ausreichen, um die relativen Stabilitäten innerhalb einer Klasse von Carbenium-Ionen mit einer Genauigkeit wiederzugeben, die der von Gasphasen-Experimenten vergleichbar ist. Daher führten wir in dieser Studie ebenfalls ab-initio-MO-Berechnungen unter Verwendung des STO-3G-Basissatzes ${ }^{13)}$ und der „Gaussian 70“-Programme ${ }^{14)}$ durch.

Die Geometrie der Methyl-substituierten Allenyl-Kationen 4-7 wurde mit Ausnahme der $\mathrm{CH}$-Bindungslängen und -winkel in den Methylgruppen vollständig optimiert; für die $\mathrm{CH}_{3}$-Gruppen wurden $\mathrm{CH}$-Bindungslängen von $1.09 \AA$ sowie Tetraederwinkel vorgegeben. Bei den Phenyl-substituierten Allenyl-Kationen 8-12 wurden nur die $C^{1} C^{2}$ - und die $C^{2} C^{3}$-Abstände optimiert. Der $C^{1} C^{2} C^{3}$-Winkel wurde gleich $180^{\circ}$ gesetzt, da bei 2-7 die Abweichung von der Linearität $0.5^{\circ}$ nie überschritt. Für die Phenylringe wurde Standardgeometrie angenommen $(\mathrm{CC}=1.40 \AA, \mathrm{CH}=1.08 \AA$, Winkel $=$ $\left(20^{\circ}\right)^{15)}$. Der dadurch eingeführte Fehler sollte klein sein, da selbst beim 1-PhenylvinylKation, in dem der Phenylrest wesentlich stärker deformiert sein muß, Optimierung des Aromaten nur mit einem Energiegewinn von $3 \mathrm{kcal} / \mathrm{mol}$ verbunden ist ${ }^{12)}$. Der $\mathrm{C}^{1}$ - Phenyl- und $\mathrm{C}^{3}$ - Phenyl-Abstand wurde für die Kationen 8 und 9 zu $1.41 \AA$ A berechnet und für die übrigen Systeme übernommen.

\section{Geometrie}

Bindungslängen: Mit 1.215 $\AA$ (Tab. 1) liegt der $C^{1} C^{2}$-Abstand des unsubstituierten Allenyl-Kations 2 zwischen der berechneten (STO-3G) Länge der Dreifachbindung des Propins $\left(\begin{array}{lll}1.170 & \AA\end{array}\right)$ und der Doppelbindung des Allens $\left.(1.288 \AA)^{18}\right)$. Die $C^{2} C^{3}$ Bindungslänge $\left(\begin{array}{ll}1.360 & \AA\end{array}\right)$ ist gegenüber der CC-Einfachbindung des Propins $(1.484 \AA)^{18)}$ verkürzt und gegenüber der Doppelbindung des Allens verlängert. Damit ist die Schreibweise des Allenyl-Kations mit einer 21/2fach-Bindung zwischen $C^{1}$ und $C^{2}$ und einer $11 / 2$ fach-Bindung zwischen $C^{2}$ und $C^{3}$ gerechtfertigt.

Methylierung an $C^{1}$ verlängert den $C^{1} C^{2}$-Abstand um 0.07-0.09 $\AA$ (Ausnahme $9 \rightarrow$ 12), während gleichzeitig der $C^{2} C^{3}$-Abstand um $0.012-0.015 \AA$ verkürzt wird. Der Einfluß von Methylgruppen an $C^{3}$ ist etwas größer: Je Methylgruppe wird $C^{1} C^{2}$ um etwa $0.01 \AA$ verkürzt und $\mathrm{C}^{2} \mathrm{C}^{3}$ um etwa $0.02 \AA$ verlängert. Phenylgruppen verschieben im gleichen Sinn, doch ist ihr Effekt größer. Methyl- wie Phenyl-Substituenten verlängern somit die benachbarte und verkürzen die entfernte Bindung des Allenylsystems. Der gleiche Effekt wurde auch bei Allyl-Kationen gefunden ${ }^{11}$. 
Tab. 1. Berechnete Gesamtenergie (a. u.) und CC-Bindungslängen ( $\dot{A})$ substituierter AllenylKationen (ab initio, STO-3G)<smiles>[R]C([R])=[C-]C([Z])([R])[C-]</smiles>

\begin{tabular}{lllllll}
\hline & $\mathrm{R}^{1}$ & $\mathrm{R}^{2}$ & $\mathrm{R}^{3}$ & Gesamtenergie & $\mathrm{C}^{1} \mathrm{C}^{2}$ & $\mathrm{C}^{2} \mathrm{C}^{3}$ \\
\hline $\mathbf{2}^{16)}$ & $\mathrm{H}$ & $\mathrm{H}$ & $\mathrm{H}$ & -113.56390 & 1.215 & 1.360 \\
$\left.\mathbf{3}^{17}\right)$ & $\mathrm{CH}_{3}$ & $\mathrm{H}$ & $\mathrm{H}$ & $-152.17978^{\mathrm{a})}$ & 1.222 & 1.348 \\
$\mathbf{4}$ & $\mathrm{H}$ & $\mathrm{CH}_{3}$ & $\mathrm{H}$ & $-152.17618^{\mathrm{a}}$ & 1.203 & 1.380 \\
$\mathbf{5 9}$ & $\mathrm{CH}_{3}$ & $\mathrm{CH}_{3}$ & $\mathrm{H}$ & -190.78708 & 1.212 & 1.365 \\
$\mathbf{6}$ & $\mathrm{H}$ & $\mathrm{CH}_{3}$ & $\mathrm{CH}_{3}$ & $\left.-190.78256^{\mathrm{a}}\right)$ & 1.196 & 1.398 \\
$\mathbf{7}$ & $\mathrm{CH}_{3}$ & $\mathrm{CH}_{3}$ & $\mathrm{CH}_{3}$ & $\left.-229.39157^{\mathrm{a}}\right)$ & 1.203 & 1.383 \\
$\mathbf{8}$ & $\mathrm{Ph}$ & $\mathrm{H}$ & $\mathrm{H}$ & -340.38512 & 1.241 & 1.325 \\
$\mathbf{9}$ & $\mathrm{H}$ & $\mathrm{Ph}_{\mathbf{1 0}}$ & $\mathrm{H}$ & -340.38984 & 1.196 & 1.410 \\
$\mathbf{1 1}$ & $\mathrm{Ph}$ & $\mathrm{CH}_{3}$ & $\mathrm{H}$ & -378.98410 & 1.230 & 1.340 \\
$\mathbf{1 2}$ & $\mathrm{CH}$ & $\mathrm{CH}_{3}$ & $\mathrm{CH}_{3}$ & -417.58286 & 1.217 & 1.359 \\
\hline
\end{tabular}

a) Lit. 9): $E(3)=-152.17940 ; E(4)=-152.17510 ; E(6)=-190.78199 ; E(7)=-229.39086$.

Konformation der Methylgruppen: Nach ab-initio-Rechnungen ist das Konformere 13 des 2-Propenyl-Kations um $0.1 \mathrm{kcal} / \mathrm{mol}$ stabiler als $14^{19)}$. Infolgedessen wurde für 1-Methyl-substituierte Allenyl-Kationen die Konformation 15 vorgegeben. Die Rotationsbarriere sollte hier noch kleiner sein als bei Vinyl-Kationen.

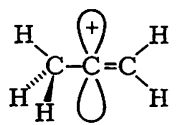

13

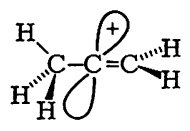

14

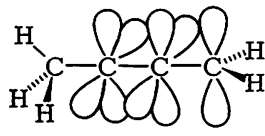

15

Die Vorzugskonformation von 3-ständigen Methylgruppen wurde am Kation 4 ermittelt. Da $4^{\prime}$ um $0.66 \mathrm{kcal} / \mathrm{mol}$ stabiler berechnet wurde als $4^{\prime \prime}$, wurde auch für 5 und $10 C_{s}$-Symmetrie vorgegeben.<smiles>[C]#CCC</smiles>

$4^{\prime}$<smiles>[C]#CCC</smiles>

$4^{\prime \prime}$<smiles>[C]#CC(C)C</smiles>

Am Kation 6 wurde gezeigt, daß die Methyl-Rotationsbarriere 3,3-dimethylsubstituierter Allenyl-Kationen kleiner als $0.5 \mathrm{kcal} / \mathrm{mol}$ ist. Die gefundene Vorzugskonformation $6^{\prime}$ des 3,3-Dimethylallenyl-Kations wurde entsprechend für die Kationen 7 und 11 übernommen.

Konformation der Phenylgruppen: Phenylgruppen an $C^{1}$ treten bevorzugt in Konjugation mit dem Allylsystem, nicht mit der ungeladenen Doppelbindung: Das planare 
Kation $8^{\prime}$ ist um $20 \mathrm{kcal} / \mathrm{mol}$ stabiler als das orthogonale Kation $\mathbf{8}^{\prime \prime}$. Dieser Unterschied entspricht der Rotationsbarriere des Phenylrests und beträgt etwa $80 \%$ des für das 1-Phenylvinyl-Kation (16) errechneten Wertes $(24.7 \mathrm{kcal} / \mathrm{mol})^{20}$.<smiles>[C]#CC=Cc1ccccc1</smiles>

$\mathbf{8}^{\prime}$<smiles>[C]C#CC#Cc1ccccc1</smiles>

$8^{\prime \prime}$<smiles>C=Cc1ccccc1</smiles>

16

Die größere Phenyl-Rotationsbarriere des 3-Phenylallenyl-Kations 9 (35 kcal/mol) ist auf den größeren LUMO-Koeffizienten an $\mathrm{C}^{3}$ der Allenyl-Kationen sowie auf das völlige Fehlen von $\pi$-Konjugation in der orthogonalen Konformation zurückzuführen.

\section{Molekülorbitale und Elektronenverteilung}

Die $\pi$-Molekülorbitale (STO-3G-Wellenfunktionen) des unsubstituierten AllenylKations 2 sind in Abb. 1 dargestellt ${ }^{21)}$. Die Orbitale rechts entsprechen dem Allylsystem. Links sind das bindende und antibindende MO der orthogonalen Doppelbindung gezeigt; die kleinen Koeffizienten an den Wasserstoffen ergeben sich durch hyperkonjugative Wechselwirkung mit den $\mathrm{CH}_{2}$-Orbitalen.

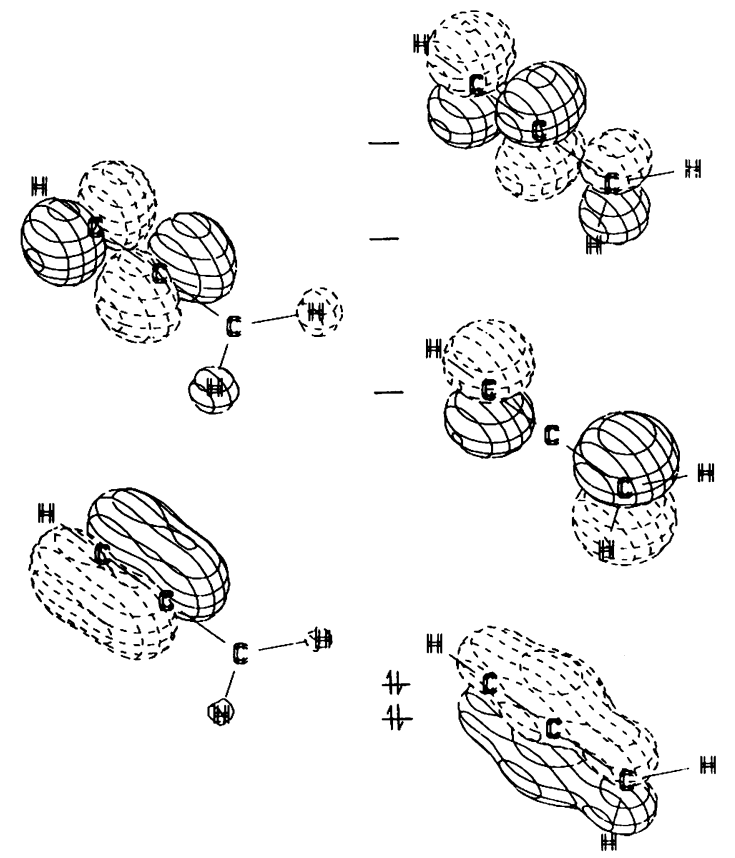

Abb. 1. $\pi$-Molekülorbitale des Allenyl-Kations 2

Das für die Reaktivität gegenüber Nucleophilen maßgebliche tiefste unbesetzte Orbital (LUMO) ist Teil des Allylsystems. Abb. 1 und Tab. 2 zeigen, daß in diesem Orbital der Koeffizient an $C^{3}$ größer ist als an $C^{1}$. Nach Tab. 2 vergrößern Phenyl- und in geringerem Maß auch Methyl-Substituenten an $C^{3}$ das Verhältnis $C^{3} / C^{1}$ der LUMO- 
Koeffizienten, während Substitution an $C^{1}$ diesen Quotienten verkleinert. Gleichfalls erhöht 3-Substitution die relative Elektronendichte an $C^{1}$, während 1-Substitution die Elektronendichte an $\mathrm{C}^{3}$ steigert. Über entsprechende Effekte an Allyl-Kationen haben wir früher berichtet ${ }^{11)}$.

Tab. 2. LUMO-Koeffizienten (obere Zahlen) und $\pi$-Ladungsdichten (Gross orbital charges, untere Zahlen) in Allenyl-Kationen

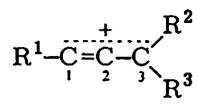

\begin{tabular}{llllrrrr}
\hline & $\mathrm{R}^{1}$ & $\mathrm{R}^{2}$ & $\mathrm{R}^{3}$ & \multicolumn{1}{c}{$\mathrm{C}^{1}$} & \multicolumn{1}{c}{$\mathrm{C}^{2}$} & $\mathrm{C}^{3}$ & $\left|\mathrm{C}^{3} / \mathrm{C}^{1}\right|$ \\
\hline $\mathbf{2}$ & $\mathrm{H}$ & $\mathrm{H}$ & $\mathrm{H}$ & -0.611 & -0.079 & 0.798 & 1.31 \\
& & & & 0.462 & 1.129 & 0.409 & 0.89 \\
$\mathbf{3}$ & $\mathrm{CH}_{3}$ & $\mathrm{H}$ & $\mathrm{H}$ & -0.626 & -0.107 & 0.776 & 1.24 \\
& & & & 0.460 & 1.128 & 0.473 & 1.03 \\
$\mathbf{4}$ & $\mathrm{H}$ & $\mathrm{CH}_{3}$ & $\mathrm{H}$ & -0.572 & -0.041 & 0.816 & 1.43 \\
& & & & 0.547 & 1.135 & 0.396 & 0.72 \\
$\mathbf{6}$ & $\mathrm{H}$ & $\mathrm{CH}_{3}$ & $\mathrm{CH}_{3}$ & -0.538 & -0.013 & 0.829 & 1.54 \\
& & & & 0.611 & 1.135 & 0.376 & 0.62 \\
7 & $\mathrm{CH}$ & $\mathrm{CH}_{3}$ & $\mathrm{CH}_{3}$ & -0.558 & -0.042 & 0.812 & 1.46 \\
& & & & 0.587 & 1.148 & 0.418 & 0.71 \\
$\mathbf{8}$ & $\mathrm{Ph}$ & $\mathrm{H}$ & $\mathrm{H}$ & -0.592 & -0.010 & 0.631 & 1.07 \\
& & & & 0.582 & 1.099 & 0.651 & 1.12 \\
$\mathbf{9}$ & $\mathrm{H}$ & $\mathrm{Ph}$ & $\mathrm{H}$ & -0.415 & -0.027 & 0.695 & 1.67 \\
& & & & 0.727 & 1.099 & 0.588 & 0.81 \\
$\mathbf{1 0}$ & $\mathrm{Ph}$ & $\mathrm{CH}_{3}$ & $\mathrm{H}$ & -0.587 & -0.067 & 0.679 & 1.16 \\
& & & & 0.585 & 1.130 & 0.581 & 0.99 \\
11 & $\mathrm{Ph}$ & $\mathrm{CH}_{3}$ & $\mathrm{CH}$ & -0.560 & -0.039 & 0.712 & 1.27 \\
& & & & 0.621 & 1.147 & 0.530 & 0.85 \\
12 & $\mathrm{CH}_{3}$ & $\mathrm{Ph}$ & $\mathrm{H}$ & -0.431 & -0.052 & 0.694 & 1.61 \\
& & & & 0.700 & 1.116 & 0.591 & 0.84 \\
\hline
\end{tabular}

\section{Energie}

Stabilisierungsenergie: Die Stabilisierung von Allenyl-Kationen durch Methyl- und Phenylgruppen kann durch die isodesmischen Reaktionen (1) - (14) definiert werden. $\Delta E$ läßt sich aus der berechneten Gesamtenergie von Ethan $(-78.30618 \text { a. u. })^{22)}$, Propan (-116.88642 a. u. $)^{18)}$ und Phenylethan $(-305.05415 \text { a. u. })^{23)}$ sowie den in Tab. 1 tabellierten Werten der Allenyl-Kationen 2-12 ermitteln.

Nach Gl. (1) bewirkt 1-Methylierung des unsubstituierten Allenyl-Kations eine Stabilisierung von $22 \mathrm{kcal} / \mathrm{mol}$. Wie erwartet, ist dieser Wert größer als beim Allyl-Kation $(17 \mathrm{kcal} / \mathrm{mol})^{11)}$, aber kleiner als beim Vinyl-Kation $(30 \mathrm{kcal} / \mathrm{mol})^{12}$. Die Stabilisierungsenergie durch 1-Methyl ist um so kleiner, je besser die positive Ladung durch 3-Substituenten delokalisiert ist (GI. 2, 3). Wie bei gesättigten Carbenium-Ionen und Vinyl-Kationen ${ }^{12)}$ ist der Effekt eines Phenylrests etwa doppelt so groß wie der eines Methylrests (Gl. 1-3 und 10-12). 


$$
\begin{aligned}
& \text { 1-Methylierung } \\
& \mathrm{H}-\mathrm{C}=\stackrel{+}{\mathrm{C}}-\ddot{\mathrm{C}}_{\mathrm{H}} \quad+\mathrm{C}_{3} \mathrm{H}_{8} \\
& \mathrm{H}-\ddot{\mathrm{C}}=\stackrel{+}{\mathrm{C}}-\stackrel{\mathrm{C}}{\mathrm{C}} \mathrm{H}\left(\mathrm{CH}_{3}\right)+\mathrm{C}_{3} \mathrm{H}_{8} \\
& \mathrm{H}-\dddot{\mathrm{C}}=\stackrel{+}{\mathrm{C}}-\mathrm{C} \mathrm{C}(\mathrm{Ph}) \quad+\mathrm{C}_{3} \mathrm{H}_{8}
\end{aligned}
$$

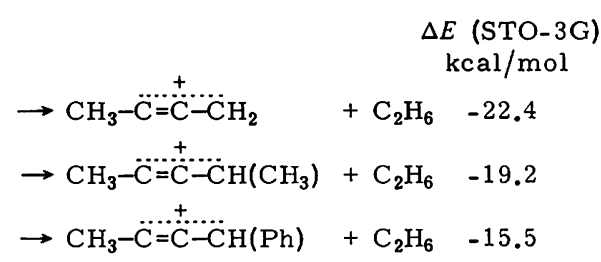

3-Methylierung

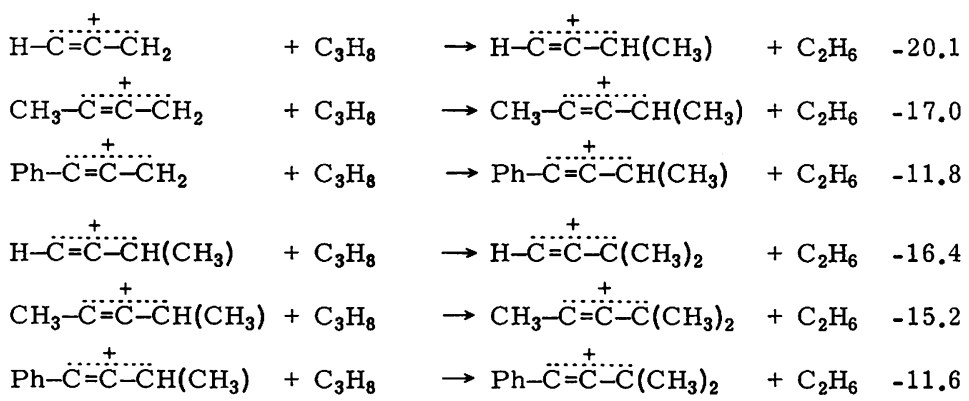

1-Phenylierung

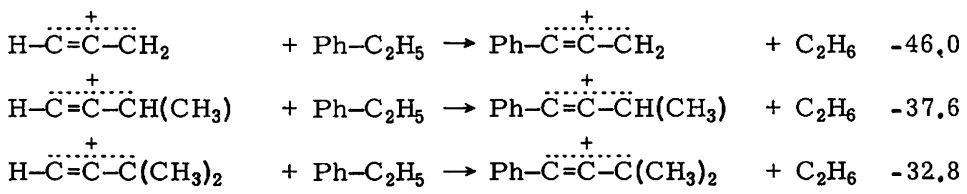

3-Phenylierung<smiles>[C]#CC(C)=CC</smiles>

$$
\begin{aligned}
& +\mathrm{Ph}-\mathrm{C}_{2} \mathrm{H}_{5} \rightarrow \mathrm{H}-\stackrel{+}{\mathrm{C}}=\stackrel{+}{\mathrm{C}}-\ddot{\mathrm{C}}_{\mathrm{C}} \mathrm{H}-\mathrm{Ph} \quad+\mathrm{C}_{2} \mathrm{H}_{6} \quad-48.9 \\
& +\mathrm{Ph}-\mathrm{C}_{2} \mathrm{H}_{5} \rightarrow \mathrm{CH}_{3}-\mathrm{C}=\stackrel{+}{\mathrm{C}}-\mathrm{C} H-\mathrm{Ph}+\mathrm{C}_{2} \mathrm{H}_{6}-42.1
\end{aligned}
$$

Einführung einer Methylgruppe in 3-Position hat einen umso größeren Effekt, je geringer die Donorfähigkeit des 1-Substituenten ist (Gl. 4-6). Die zweite 3-Methylgruppe stabilisiert um einen etwas geringeren Betrag (Gl. 7-9). 3-Phenylreste haben einen mehr als doppelt so großen Effekt wie entsprechende Methylgruppen (Gl. 13, 14).

Zunächst überrascht der Befund, daß 1-Methylierung eine größere Stabilisierung bewirkt als 3-Methylierung (Gl. 1 und 4), da aufgrund des größeren LUMO-Koeffizienten an $C^{3}$ das Gegenteil zu erwarten wäre. Nach der hier gewählten Definition der Stabilisierungsenergien reflektieren diese Zahlen aber außer der Stabilisierung der positiven Ladung zusätzlich den Energieunterschied, um den 2-Butin stabiler ist als 1-Butin (nach STO-3G $7.1 \mathrm{kcal} / \mathrm{mol}^{24)}$ ). Berücksichtigt man diesen Betrag, so erhält man die erwartete Reihenfolge. Phenyl-Substitution hat in Position 3 einen größeren Effekt als in Position 1. Wiederum dürfte die relative Energie der korrespondierenden Kohlenwasser- 
stoffe dafür verantwortlich sein, daß nach dieser Definition der Unterschied relativ klein ist.

Berechnung von Bildungswärmen: Aus den berechneten $\Delta E$-Werten und den experimentellen $\Delta H_{\mathrm{f}}^{\mathrm{o}}$-Werten des Allenyl-Kations $2^{25)}$, Ethan $(-20.2 \mathrm{kcal} / \mathrm{mol})$, Propan $(-24.8 \mathrm{kcal} / \mathrm{mol})$ und Ethylbenzol $(+7.1 \mathrm{kcal} / \mathrm{mol})^{26)}$ lassen sich nun die Bildungswärmen der Allenyl-Kationen 3-12 berechnen (Tab. 3). Außer für 2 sind auch für die Kationen $3(255)^{27)}, 4(257)^{27)}$ und $6(\approx 234)^{28)}$ experimentelle Bildungswärmen bekannt. Die hervorragende Übereinstimmung kann sowohl als Beweis für die Zuverlässigkeit dieser Abschätzungen wie auch als Bestätigung der internen Konsistenz des experimentellen Datensatzes angesehen werden. Eine früher angegebene Bildungswärme von $254 \mathrm{kcal} / \mathrm{mol}$ für Kation $2^{29)}$ läßt sich aufgrund dieser Berechnungen mit Sicherheit ausschließen.

Tab. 3. Berechnete Bildungswärmen $\left(\Delta H_{\mathrm{f}}^{\mathrm{o}}\right)^{\text {a) }}$ der Allenyl-Kationen 2-12 $(\mathrm{kcal} / \mathrm{mol})$

\begin{tabular}{rrrrrrrr}
\hline $\mathbf{2}$ & $281^{\mathrm{b})}$ & $\mathbf{5}$ & 232.5 & $\mathbf{8}$ & 262.3 & $\mathbf{1 1}$ & 229.8 \\
$\mathbf{3}$ & 254.0 & $\mathbf{6}$ & 235.3 & $\mathbf{9}$ & 259.4 & $\mathbf{1 2}$ & 239.2 \\
$\mathbf{4}$ & 256.3 & $\mathbf{7}$ & 212.7 & $\mathbf{1 0}$ & 246.0 & & \\
\hline
\end{tabular}

a) Dezimalstellen werden mitgeführt, um Fortpflanzung von Rundungsfehlern zu vermeiden. -

b) Referenzverbindung (Lit. ${ }^{25)}$ ).

\section{Konsequenzen für das Additions- und Cycloadditionsverhalten von Allenyl-Kationen}

Wie bereits aus experimentellen ${ }^{1-5)}$ und früheren theoretischen Untersuchungen ${ }^{7-9)}$ gefolgert, sind beide Resonanzstrukturen 1a und 1b am Grundzustand von AllenylKationen beteiligt. Substitution an $C^{1}$ begünstigt die Allen-, Substitution an $C^{3}$ die Alkin-Grenzstruktur.

Reaktionen mit n-Nucleophilen: Der Angriff harter Nucleophile auf Allenyl-Kationen sollte durch die Ladungsverteilung in $\mathbf{2 - 1 2}$ determiniert werden. Sieht man von den 1 -monosubstituierten Kationen $\mathbf{3}$ und $\mathbf{8}$ ab, ist nach Tab. 2 die positive Ladung an $\mathrm{C}^{3}$ stets größer als an $C^{\prime}$. Damit ist zu erklären, daß Allenyl-Kationen von n-Nucleophilen primär an $\mathrm{C}^{3}$ angegriffen werden, so daß unter kinetisch kontrollierten Bedingungen vorwiegend Propargylderivate entstehen (Gl. 15a). Dagegen ist die vielfach beobachtete Bildung von Allenylderivaten (Gl. 15b) im allgemeinen auf thermodynamische Produktkontrolle zurückzuführen ${ }^{30)}$.

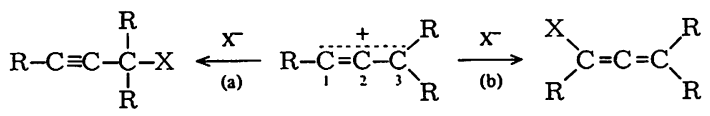

Reaktionen mit $\pi$-Nucleophilen: Nach der Störungs-MO-Betrachtung sollten $\pi$ Systeme (weiche Nucleophile) ebenfalls bevorzugt an $\mathrm{C}^{3}$ angreifen (Gl. 16a), weil nach Tab. 2 der LUMO-Koeffizient an dieser Position am größten ist. Natürlich gilt diese Erwartung nur, wenn $\mathrm{C}^{3}$ sterisch nicht stark abgeschirmt ist; ist dies der Fall, muß auch mit $C^{1}$-Angriff gerechnet werden. 


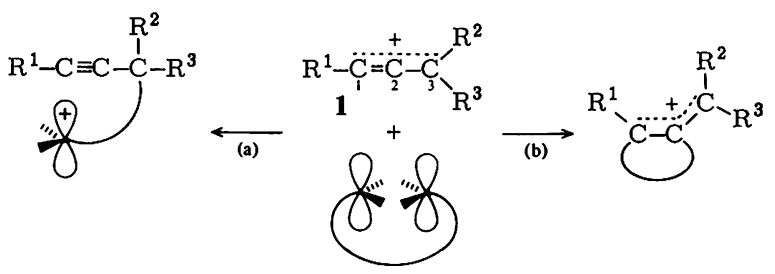

Die PMO-Methode ist nur auf Reaktionen mit frühem Übergangszustand anwendbar. Führt man nun im Allenyl-Kation 1 zunehmend elektronenliefernde Substituenten ein, was nach Abschnitt 4 zur Stabilisierung von 1 führt, wird bei konstant gehaltenem Nucleophil die Reaktion (16a) zunehmend weniger exotherm. Der Übergangszustand, der zunächst Edukt-ähnlich ist (frontier orbital control), wird allmählich Produkt-ähnlich. Reaktionsweg (16a), bei dem der stabilisierende Effekt der Substituenten $\mathrm{R}^{1}-\mathrm{R}^{3}$ verlorengeht, wird dabei unattraktiver. Dagegen gewinnt Reaktionsweg (16b) an Bedeutung, weil hier die Substituenten $R^{1}-R^{3}$, die zunächst das Allenyl-Kation stabilisieren, auch im Produkt einen stabilisierenden Effekt ausüben können.

Es ist daher zu erwarten, daß wenig stabilisierte Allenyl-Kationen nach Gl. (16a) reagieren, und daß bei zunehmender Donorfähigkeit von $R^{1}-R^{3}$ Weg (16a) von (16b) abgelöst wird. Als Grenzfall des Reaktionstypus (16b) lassen sich die Diels-Alder-Reaktionen von Alkinylamidium-Ionen mit Cyclopentadien (Gl. 17) ${ }^{31)}$ wie auch die LewisSäure-katalysierten Additionen von Propiolsäureestern an Olefine (Gl. 18) auffassen ${ }^{32}$.
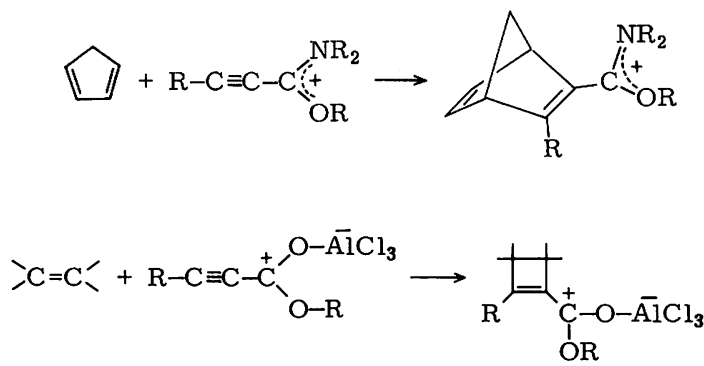

Es läßt sich nicht generell voraussagen, bei welchem Substitutionsgrad der Wechsel zwischen Reaktionsweg (16a) und (16b) erfolgt, da dies außer von $R^{1}-R^{3}$ auch vom Nucleophil abhängig ist.

Wir danken Herrn Prof. P. v. R. Schleyer für Diskussionen und die zur Verfügung gestellte Rechenzeit, der Deutschen Forschungsgemeinschaft und dem Fonds der Chemischen Industrie für finanzielle Unterstützung.

1) H. G. Richey und J. M. Richey in Carbonium Ions (Herausg. G. A. Olah und P. v. R. Schleyer), Bd. II, Kap. 21, Wiley Interscience, New York 1970.

2) G. Modena und $U$. Tonellato, Adv. Phys. Org. Chem. 9, 185 (1971).

3) P. J. Stang, Prog. Phys. Org. Chem. 10, 205 (1973).

4) M. Hanack, Acc. Chem. Res. 9, 364 (1976).

5) P. J. Stang, Z. Rappoport, M. Hanack und L. R. Subramanian, Vinyl Cations, S. 246-270, 486 - 489, Academic Press, New York 1979. 
6) H. Mayr, Habilitationsschrift, Univ. Erlangen 1980. Siehe auch nachstehende Publikationen und dort zitierte Arbeiten.

7) D. Mirejovsky, W. Drenth und F. B. van Duijneveldt, J. Org. Chem. 43, 763 (1978).

8) D. Mirejovsky, W. Drenth und F. B. van Duijneveldt, Rec. Trav. Chim. Pays-Bas 98, 388 (1979).

9) M. Dorado, O. Mó und M. Yánez, J. Am. Chem. Soc. 102, 947 (1980).

10) K. Raghavachari, R. A. Whiteside, J. A. Pople und P. v. R. Schleyer, J. Am. Chem. Soc. 103, 5649 (1981).

11) H. Mayr, W. Förner und P. v. R. Schleyer, J. Am. Chem. Soc. 101, 6032 (1979); 102, 3663 (1980).

12) H. Mayr, R. Schneider, D. Wilhelm und P. v. R. Schleyer, J. Org. Chem. 46, 5336 (1981).

13) W. J. Hehre, R. Ditchfield, R. F. Stewart und J. A. Pople, J. Chem. Phys. 51, 2647 (1969).

14) W. J. Hehre, W. A. Lathan, R. Ditchfield, M. D. Newton und J. A. Pople, QCPE Programm Nr. 236, Indiana University, Bloomington, Indiana.

15) J. A. Pople und M. Gordon, J. Am. Chem. Soc. 89, 4253 (1967).

16) L. Radom, P. C. Hariharan, J. A. Pople und P. v. R. Schleyer, J. Am. Chem. Soc. 98, 10 (1976).

17) J. Chandrasekhar und P. v. R. Schleyer, unveröffentlichte Ergebnisse.

18) L. Radom, W. A. Lathan, W. J. Hehre und J. A. Pople, J. Am. Chem. Soc. 93, 5339 (1971).

19) L. Radom, P. C. Hariharan, J. A. Pople und P. v. R. Schleyer, J. Am. Chem. Soc. 95, 6531 (1973).

20) Y. Apeloig, P. v. R. Schleyer und J. A. Pople, J. Org. Chem. 42, 3004 (1977).

21) Plotting-Programm: $W$. L. Jorgensen, QCPE Programm Nr. 340, Indiana University, Bloomington, Indiana.

22) W. A. Lathan, W. J. Hehre und J. A. Pople, J. Am. Chem. Soc. 93, 808 (1971).

23) Eigene Berechnungen mit Standardgeometrie.

24) W. J. Hehre und J. Pople, J. Am. Chem. Soc. 97, 6941 (1975).

25) F. P. Lossing, Can. J. Chem. 50, 3973 (1972).

26) J. D. Cox und G. Pilcher, Thermochemistry of Organic and Organometallic Compounds, Academic Press, New York 1970.

27) Privatmitteilung von Prof. Lossing an Prof. Schleyer, Januar 1977.

28) F. P. Lossing und J. C. Traeger, Int. J. Mass Spectrom. Ion Phys. 19, 9 (1976).

29) $K$. W. Egger und A. T. Cocks, Helv. Chim. Acta 56, 1516 (1973).

30) T. L. Jacobs und D. M. Fenton, J. Org. Chem. 30, 1808 (1965).

31) J. S. Baum und H. G. Viehe, J. Org. Chem. 41, 183 (1976).

32) B. B. Snider, D. J. Rodini, R. S. E. Conn und S. Sealfon, J. Am. Chem. Soc. 101, 5283 (1979). 